\title{
Smarte Konzepte für smarte Sensoren
}

\section{Einfache und schnelle Nutzung aller Funktionen und Daten}

\begin{abstract}
Der volle Funktionsumfang eines modernen Feldgeräts korreliert mit mehreren hundert Geräteparametern. Für eine schnelle Inbetriebnahme eines Geräts ist diese Vielzahl an Parametern aber meist nicht erforderlich, für die notwendigen Basisfunktionen des Feldgeräts reichen einige wenige aus. Diese werden im Beitrag als Ergebnis eines NamurAd-hoc-Arbeitskreises identifiziert und als Namur-Standardgeräteparameter bezeichnet. Weiterhin wird vorgeschlagen diese Festlegungen der Standardgeräteparameter im Rahmen der Umsetzung der Geräteintegrationstechnologie FDI für alle Feldgeräte in einheitlicher Form als Teil der FDI Device Packages zu verwenden. Ferner werden die Möglichkeiten diskutiert, wie die verfügbare Sensorinformation übertragen und in die Leittechnik beziehungsweise Hostsysteme integriert werden kann. Dazu werden aktuelle Entwicklungen im Bereich Übertragungstechnologien diskutiert und Erwartungen formuliert. Bei der Geräteintegration wird ebenso der aktuelle Status in Bezug auf FDI als Geräteintegrationstechnologie dargestellt und wichtige Erfolgsfaktoren für die Akzeptanz der Technologie werden aus Anwendersicht definiert.
\end{abstract}

\section{SCHLAGWÖRTER Standardisierung / Geräteparameter / FDI / Ethernet / FDI device package}

\section{Smart concepts for smart sensors - Flexible, uniform use of all functions and data}

Full functionality of a modern field device may involve hundreds of device parameters. But rapid commissioning of the field device usually only requires basic functions with a few parameters, which have been specified by a NAMUR ad hoc working group as NAMUR standard device parameters. In the context of realization of field device integration technology FDI, we propose applying these specifications in a unified way as an integral part of the FDI device packages. Further, we discuss how the available information from a sensor could be transferred to the control systems and integrated. Current developments for transferring information within the process industry are discussed. Regarding device integration, the current status of FDI technology is shown and important factors are defined for the acceptance of FDI by customers. 
ARMIN BRUCKER, OLIVER WEIGEL, BASF

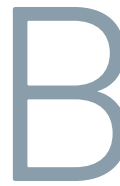

ei den Feldgeräten ist festzustellen, dass aufgrund der immer weiter steigenden Funktionalitäten auch immer mehr zusätzliche $\mathrm{Ge}$ räteparameter erforderlich sind, um diese Funktionen zu aktivieren beziehungsweise an die Applikation anzupassen. Mittlerweile haben beispielsweise Coriolis-Massedurchflussmesser rund 700 Geräteparameter und das mit steigender Tendenz.

Über die originäre Messgröße hinaus können Feldgeräte inzwischen weitere Prozessdaten und/oder gerätespezifische Daten zur Verfügung stellen. Für das Beispiel Coriolis-Massedurchflussmesser sind das Dichte, Viskosität und Temperatur und interne Gerätedaten wie Rohrsteifigkeit und Schwingungsdämpfung. Diese Daten sind wichtig für das Assetmanagement und die Instandhaltung, sie führen aber zu einer immer weiter steigenden Datenflut vom Feldgerät hin zu den übergeordneten Ebenen der Prozessautomation. Für diese Daten werden entsprechende performante Übertragungstechnologien sowie Technologien zur einfachen und langzeitstabilen Feldgeräteintegration benötigt. Die entsprechenden Technologien sind die Voraussetzung für die weitere Nutzung in überlagerten Ebenen der Prozessautomation.

\section{SMARTE SENSOREN}

Komplexe Feldgeräte im Auslieferungsstand so von den Herstellern zu erhalten, dass eine schnelle Inbetriebnahme ermöglicht wird, ist ein langgehegter Wunsch der Instandhalter und Projektierer. Es ist eine Herausforderung, dies herstellerübergreifend für alle Messverfahren und in einer einheitlichen Form zu erreichen. Diese Vereinfachung der Geräteparametrierung muss eine Eigenschaft von smarten Sensoren sein. Smarte Feldgeräte zeichnen sich durch eine schnelle Inbetriebnahme der Basisfunktion Messen der Prozessgröße aus. Für den Anwender sollen die Geräte in einheitlicher Form parametrierbar sein, wobei im Auslieferungszustand die mit der Messaufgabe verbundenen Geräteparameter eine einheitliche Bezeichung und die gleichen Werte haben müssen. Die herstellerspezi- fischen Zusatzfunktionen, mit denen sich die Hersteller voneinander im Wettberwerb differenzieren, sind für eine schnelle Inbetriebnahme allerdings von untergeordneter Bedeutung. Solche Funktionalitäten sind mit ganz unterschiedlichen Geräteparametern und Parametereinstellungen verbunden und lassen sich auch künftig nicht standardisieren. Maßstab für das smarte Feldgerät ist eine Herangehensweise wie sie vom Automobilbau her bekannt ist: Grundsätzliche Dinge, wie Gaspedal, Bremse, Blinker, die Anordnung der Schaltelemente im Auto, sind standardisiert, um das Fahrzeug möglichst schnell startbereit zu machen, Zusatzfunktionen, wie Fahrwerkeinstellungen, bedürfen eines Blicks in die Bedienungsanleitung und Zeit.

\subsection{Namur-Standardgeräteparameter}

Um diese Herausforderung für Feldgeräte anzunehmen und allgemein akzeptierte Lösungen zu finden, wurde 2010 ein Namur-Ad-hoc-Arbeitskreis „Geräteparameter" gegründet. Hersteller und Anwender haben dabei nach der 80:20-Regel für den Standardanwendungsfall Funktionen und die dazugehörenden Geräteparameter identifiziert und darüberhinaus auch die Default-Werte für diese Geräteparameter festgelegt. Betrachtet wurden dabei Durchfluss-, Druck-, Temperatur- und Standmessgeräte sowie Stellungsregler.

Es zeigte sich schnell, dass für das Messen der Prozessgröße selbst wenige Parameter erforderlich sind. Die eingangs erwähnten rund 700 Parameter sind größtenteils mit einer Fülle von Zusatzfunktionen verknüpft, die sehr oft sogar nur auf speziellen Kundenwunsch applikationsabhängig realisiert wurden und so in den allermeisten Anwendungsfällen gar nicht benötigt werden. Bei der Diskussion über die Basisfunktionen und die zugeordneten Geräteparameter zeigten sich deutliche Unterschiede von Hersteller zu Hersteller bei der Bezeichnung und Namensgebung. Um Fehlinterpretationen zu vermeiden und zur Standardisierung wurden deshalb für die betrachteten Parameter Begriffe und Kennzeichnungen der IEC 61987 [1] und 61360 [2] verwendet. 


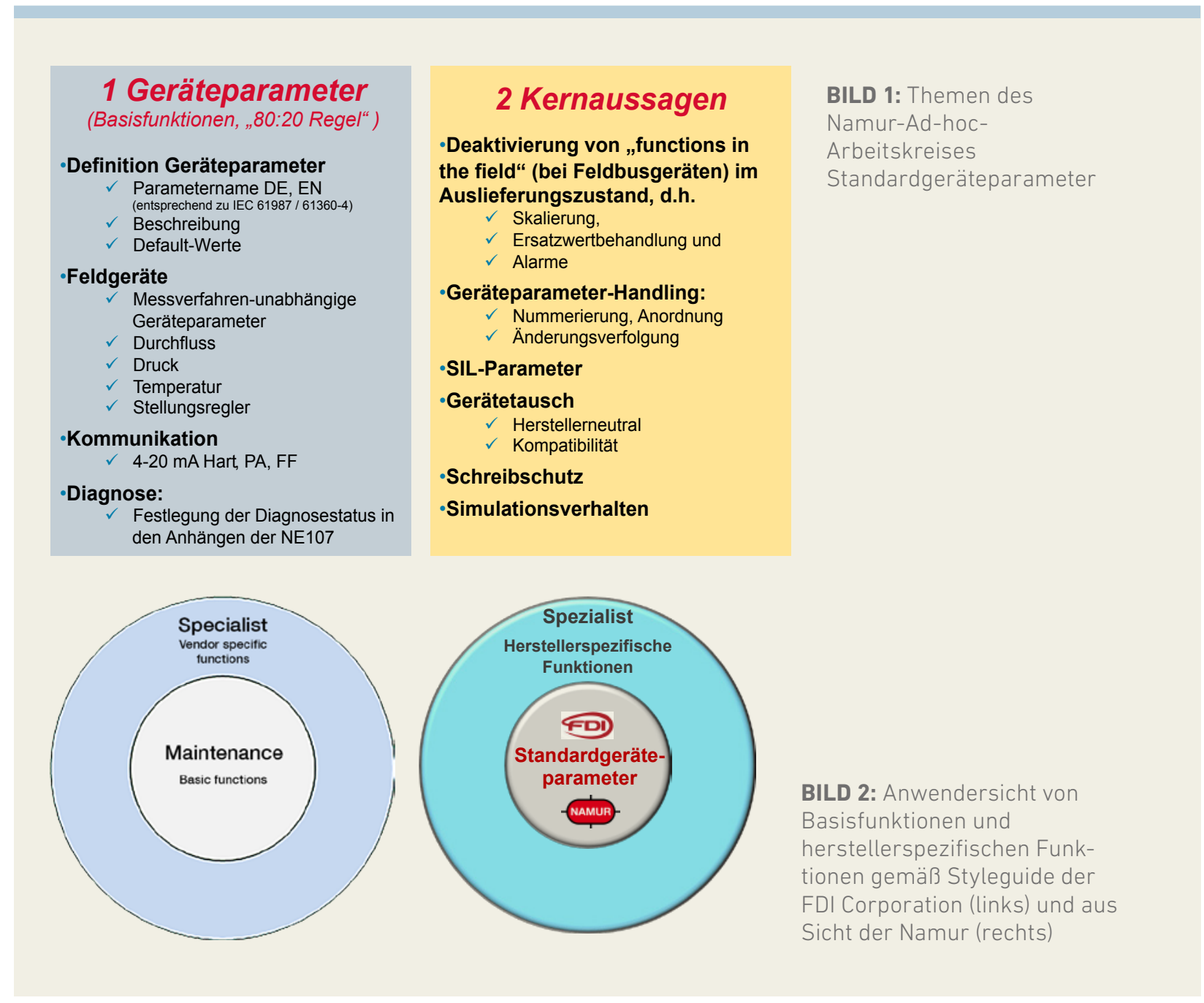

Interessanterweise stellte sich bei den Diskussionen heraus, dass die für eine schnelle Inbetriebnahme notwendigen Geräteparameter gar nicht messverfahrenspezifischer Natur sind: 18 Geräteparameter sind universell für jedes Messverfahren verwendbar. Nur wenige Parameter tragen dem speziellen Messverfahren Rechnung. Sie machen bei den betrachteten fünf Gerätearten in Summe nur 20 zusätzliche Parameter aus. Damit reichen für die schnelle Inbetriebnahme der Basisfunktionen eines Feldgeräts schon 20-25 Parameter aus, die Namur-Standardparameter. Bei den Festlegungen wurden analoge (4$20 \mathrm{~mA}$ ) Geräte und Feldbusgeräte (FF, PA) berücksichtigt. Generell wurde die mit Feldbusgeräten mögliche Verlagerung von Funktionalitäten ins Feldgerät, wie zum Beispiel Skalierung, Ersatzwertbehandlung und damit verbundene Alarme, nicht betrachtet, da diese Geräteeigenschaften von den allermeisten Anwen- dern im Prozessleitsystem realisiert werden. Alle damit verbundenen Geräteparameter sind deshalb im Auslieferungszustand so einzustellen, dass diese Funktionen deaktiviert sind und damit im Ausgang ein unmodifizierter Messwert vorliegt.

Bei den Betrachtungen zum Standardeinsatzfall wurden im Arbeitskreis ebenso Themen wie die Identifizierung von Geräteparametern mit Relevanz für PLT-Sicherheitsfunktionen (zum Beispiel Messwertdämpfung und T90-Zeit), die Änderungsverfolgung und der Schreibschutz, das Geräteparameter-Handling, das Simulationsverhalten und Gerätediagnosen bearbeitet. Beim Thema Diagnose stand vor allem die Zuordnung der in den messaufgaben- und gerätespezifischen Anhängen der NE 107 aufgeführten Diagnosen zu den Namur-Status im Fokus. Im Bild 1 sind alle Themenschwerpunkte des Namur-Ad-hoc-Arbeitskreises Geräteparameter zusammengefasst. Die Ar- 
beitsergebnisse hierzu sollen in die Revisionen der NE 131 Namur-Standardgerät - Anforderungen an Feldgeräte für Standardanwendungen [3], der NE 107 Selbstüberwachung und Diagnose von Feldgeräten [4] und der NE 43 Vereinheitlichung des Signalpegels für die Ausfallinformation von Digitalen Messumformern mit analogem Ausgangssignal [5] einfließen.

Die im Arbeitskreis angestrebte Standardisierung von typischen Gerätefunktionen und deren Parametern ist nicht neu, von Feldbusseite sind Transducer Profiles [6, 7], bei Hart-Geräten die Device Families and Command Specifications [8] mit den entsprechenden Spezifikationshandbüchern veröffentlicht. Bei diesen Konzepten der Geräteparametrierung und Geräteintegration waren allerdings die Anwender bei der Erarbeitung der Festlegungen nur wenig eingebunden, ferner ist diese konzeptionelle Umsetzung beim Anwender nur wenig bekannt. Noch entscheidender ist aber, dass es für den Feldgerätehersteller keine Verpflichtung gibt, diese Standardfunktionen und verknüpften Parameter in dieser Form anzubieten. Vielmehr werden von Herstellerseite die herstellerspezifischen Zusatzfunktionen in den Vordergrund gerückt und nicht klar zwischen diesen und den Standardparametern differenziert.

\subsection{Field Device Integration mit Standardgeräteparameter}

Mit dem Konzept Field Device Integration (FDI) zeichnet sich derzeit ein neuer Ansatz der Feldgeräteintegration ab. Unter dem Dach der FDI Cooperation haben sich die FDT Group, Fieldbus Foundation, Hart Communication Foundation, Profibus \& Profinet und OPC Foundation zusammengeschlossen, um herstellerübergreifend und in einheitlicher Form die Feldgeräteintegration zu gewährleisten. Im Styleguide der FDI [9] wird zwischen unterschiedlichen Rollen und Anforderungen an die Geräteparametrierung unterschieden: den Basisfunktionen des Geräts für den Personenkreis der Instandhaltung und den herstellerspezifischen Funktionen für den Spezialisten, siehe Bild 2a. Den unterschiedlichen Funktionen entsprechend werden Geräteparameter zugeordnet, wobei die Parameter, die die Basisfunktionen beschreiben, als core parameter bezeichnet werden. Dieser Ansatz zur Differenzierung der unterschiedlichen Gerätefunktionen ist identisch zu dem des NamurAd-hoc-Arbeitskreises Geräteparameter!

Weiter steht im Styleguide für die core parameter: "The default definition of the core variable/parameter attributes for Maintenance shall be provided by the manufacturer based on general customer requirements (e.g. NAMUR recommendations).” Somit können die Namur-Standardgeräteparameter eins zu eins von der FDI für die dort mit core parameter be- zeichneten Geräteparameter übernommen werden, siehe Bild 2b. Sie sind der standardisierte Teil der Gerätebeschreibung, die der schnellen und einfachen Geräteinbetriebnahme dient, weitere allgemeine oder herstellerspezifische Funktionen sind weitere Bestandteile der Gerätebeschreibung.

Mit der flächendeckenden Einführung von Gerätebeschreibungen nach dem FDI-Konzept, den FDI-Packages, besteht die Aussicht, in Zukunft für alle Feldgeräte eine herstellerneutrale und einheitliche Parametrieroberfläche für die Basisfunktionen und deren Parameter zu erhalten. Der Wunsch der Anwender nach einem einheitlichen Look-andfeel bei der Gerätekonfiguration ist damit in greifbare Nähe gerückt. Der Anwender muss sich bei der Inbetriebnahme nicht um Hunderte von Geräteparametern kümmern, sondern bekommt die notwendigen Standardgeräteparameter für die Basisfunktionen für alle Gerätearten in einheitlicher Form dargestellt. Diese Konzentration auf die wesentlichen Geräteparameter und die Belegung dieser Parameter mit Werten für den Standardanwendungsfall, schon im Auslieferungszustand, ermöglicht die schnelle Inbetriebnahme des Geräts oder vereinfacht einen notwendigen Geräteaustausch. Da sich die Standardisierung auf die Basisfunktionen beziehungsweise die dazugehörenden Geräteparameter beschränkt, ist dieses Konzept ebenso bei Geräteweiterentwicklungen revisionssicher. Spezielle Gerätefunktionen sind mit zusätzlichen Geräteparametern verknüpft, vervollständigen die Gerätebeschreibung des Feldgeräts und erfordern wegen ihrer Herstellerspezifika vertiefte Kenntnisse des Geräteexperten. Die Feldgerätehersteller sind aufgefordert, ihre Geräte künftig mit den FDI-Gerätebeschreibungen (FDI-Packages) mit den NamurStandardgeräteparametern für die schnelle Nutzung der Basisfunktionen auszuliefern.

\section{SMARTE KONZEPTE}

\subsection{Feldgeräteintegration}

Basierend auf den definierten Standardgeräteparametern und den spezifischen Gerätefunktionen kann der Feldgerätehersteller ein FDI-Package erstellen. Das FDIPackage beinhaltet die gerätespezifische Information, um das Feldgerät in entsprechende Hostsysteme, wie zum Beispiel ein Prozessleitsystem, einzubinden.

Das FDI-Package, siehe Bild 3, besteht unter anderem aus einer Electronic Device Description Language (EDDL), die

- die Gerätefunktionalität beschreibt und

- eine einfache grafische Bedienoberfläche (User Interface Descrption/UID) im Host ermöglicht. 

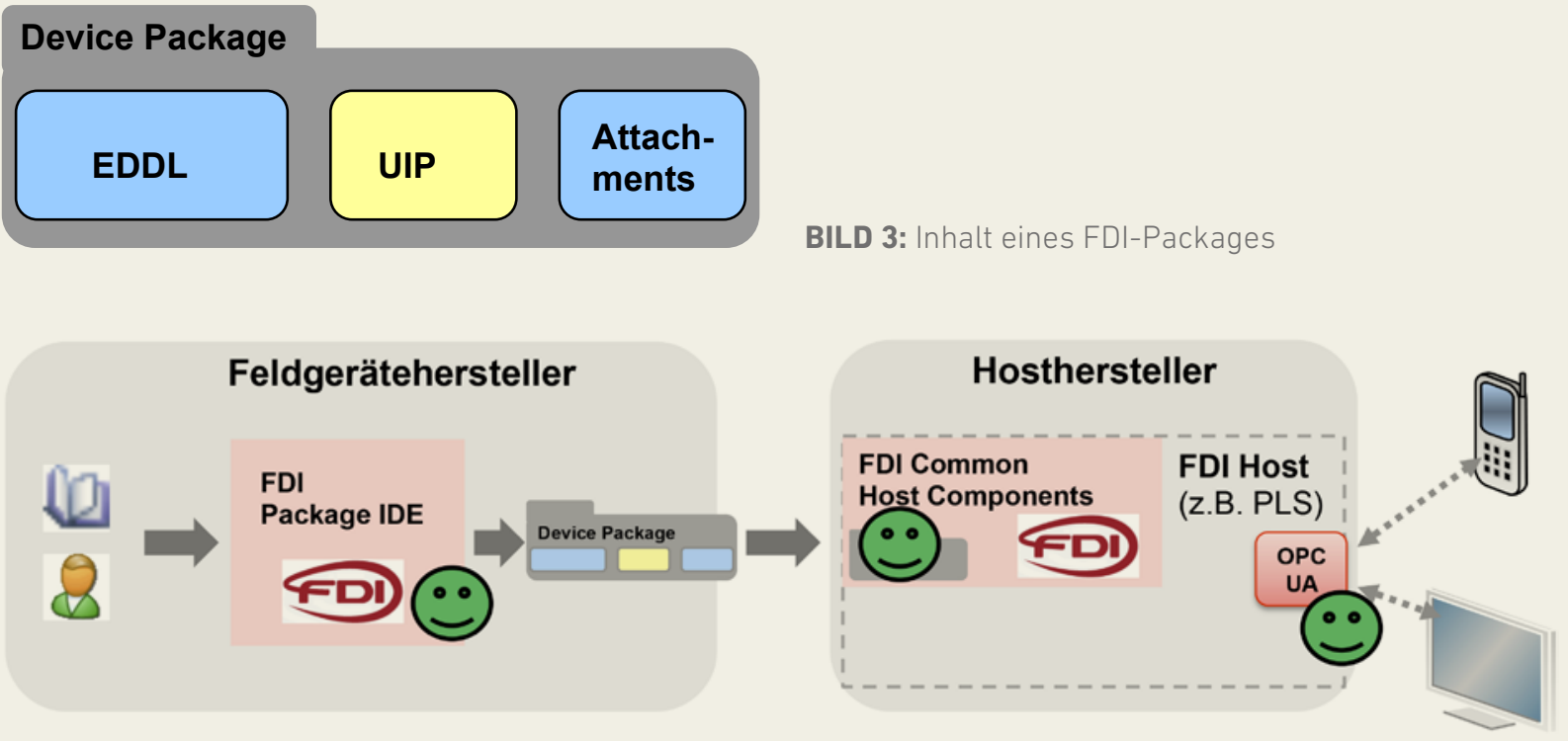

BILD 4: Geräteintegration mittels FDI

$\begin{array}{cll}\text { Information zur } & \text { Zusatzinformation } & \text { Gerätediagnosen (NE107) } \\ \text { Prozessführung } & \text { über den Prozess } & \text { - Statussignale } \\ & & \text { - Details }\end{array}$
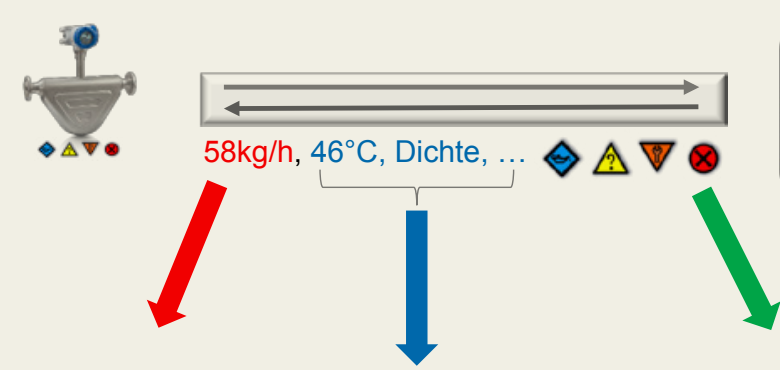

Analog: $4-20 \mathrm{~mA}$

Weitere Stromausgänge und/oder Hart

Feldbus: Messwert zyklisch weitere Messwerte zyklisch

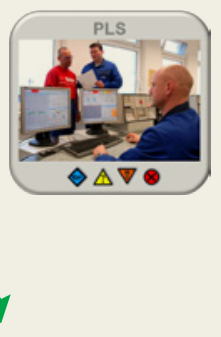

Hart (zyklisch) \& azyklisch

zyklisch \& azyklisch
BILD 5: Darstellung der übertragenen Information eines Feldgeräts
Darüber hinaus gibt es noch folgende Komponenten:

- eine grafisch aufwendigere Benutzerschnittstelle mittels einer Programmiersprache, die auch Zusatzfunktionen (User Interface Plugin/UIP) realisieren kann,

- Anhänge, wie zum Beispiel bei Profibus die Gerätestammdatei (GSD) oder Dateianhänge wie das Handbuch.
Das Hostsystem importiert das entsprechende FDI-Package und interpretiert die im Paket enthaltenen Komponenten beziehungsweise führt die entsprechenden Funktionen aus. Bei der Interaktion zwischen Feldgeräte- und Hosthersteller ist die zentrale Schnittstelle das FDI-Package. Die FDI-Spezifikation legt fest wie das entsprechende FDI-Package vom Feldgerätehersteller zu erstellen und auf der anderen Seite vom Hostsystemhersteller zu interpretieren ist, siehe Bild 4. 
Zur Vereinfachung der Erstellung der FDI-Packages und der Interpretation in den Hostsystemen wurden im Rahmen des FDI-Projektes erstmalig entsprechende Softwarekomponenten entwickelt und weiterhin zentral gepflegt. Diese Softwarekomponenten sind

- die FDI-Package IDE zur Erstellung der FDI-Packages durch den Feldgerätehersteller und

- die FDI Common Host Components zur Interpretation und Ausführung des FDI-Packages in einem Hostsystem.

Die FDI-Package IDE und die FDI Common Host Components setzen somit die FDI-Spezifikation um und sie sollen sicherstellen, dass jedes FDI-Package in jedem FDI-Host gleich funktioniert. Dadurch sind die Entwicklungsumgebung FDI-Package IDE und die Hostkomponente FDI Common Host Components elementarer Bestandteil des FDI-Standards.

Der Einsatz der entsprechend standardisierten und gepflegten Softwarekomponenten durch alle Feldgerätehersteller und Hostsystemhersteller hat den Vorteil, dass eine einheitliche Interpretation der FDI-Spezifikation erfolgt. Dadurch ist eine unterschiedliche Interpretation der FDI-Spezifikation praktisch ausgeschlossen und ebenso damit einhergehende Fehler und Inkompatibilitäten zwischen einem FDI-Package und einem Hostsystem.

Darüber hinaus lassen sich Programmierfehler in der Regel schneller erkennen als bei individuellen Lösungen, da die standardisierten Komponenten wesentlich häufiger und in verschiedensten Umgebungen eingesetzt werden

Sollte ein Feldgeräte- oder ein Hostsystemhersteller nicht die zentral entwickelten und gepflegten Softwarekomponenten einsetzen, muss dieser Hersteller einen sehr aufwendigen Softwareentwicklungsprozess durchführen und unterhalten, um Inkompatibilitäten zum FDI-Standard zu vermeiden. Darüber hinaus wird sich bei Inkompatibilitäten beziehungsweise Problemen, zum Beispiel zwischen einem FDIPackage, das mittels der zentral gepflegten FDI-Package IDE erstellt wurde, und einem Hostsystem, das herstellerspezifisch entwickelte Softwarekomponenten zur Interpretation des FDI-Standards einsetzt, immer der Hersteller mit den eigenentwickelten Softwarekomponenten als Erstes gegenüber dem Anwender rechtfertigen müssen.

Da die standardisierten Softwarekomponenten zur Verfügung stehen und eine kontinuierliche Pflege durch die FDI Cooperation beziehungsweise deren Nachfolger erfolgt, sollten diese Softwarekomponenten eingesetzt werden, da diese faktisch die Umsetzung des FDI-Standards darstellen. Für die Anwender ist der Einsatz der zentral gepflegten Softwarekomponenten zur langfristigen Sicherstellung der Kompatibilität elementar und somit ein wichtiger Erfolgsfaktor für FDI.

\section{2 Übertragungstechnologien}

Neben der Feldgeräteintegration spielt die zugehörige Übertragungstechnologie eine Schlüsselrolle zur Nutzung der Feldgerätedaten. Zur Zeit ist es die Aufgabe der Übertragungstechnologie primär, die benötigte Information zur Prozessführung, in der Regel den Messwert, zuverlässig zum Leitsystem zu übertragen. Hierfür werden etablierte Technologien, wie 4-20mA mit und ohne Hart, sowie Feldbustechnologien, wie Profibus PA [10], eingesetzt. Darüber hinaus bieten Feldgeräte aber noch eine Vielzahl weiterer möglicher Zusatzinformation über den Prozess sowie Diagnosedaten über ihren eigenen Zustand. Diese Zusatzinformation kann mit den genannten etablierten Feldbustechnologien zwar übertragen werden, allerdings gibt es Limitierungen der Bandbreite beziehungsweise eine nur teilautomatisierte Übertragung und Verarbeitung der Zusatzinformation, siehe Bild 5.

Damit die genannte Zusatzinformation für Auswertungen beziehungsweise wie von [11] gefordert Daten als Infrastrukturdienst zur Verfügung stehen, ist der Einsatz anderer Übertragungstechnologien als bisher notwendig. Eine Möglichkeit ist der Einsatz von Ethernet.

\subsection{Ethernet als Übertragungstechnologie in der Prozessindustrie}

Die Ethernet-Technologie ist aus dem IT-Umfeld bekannt und stellt einen weltweiten Standard für die Vernetzung von Komponenten dar. Allerdings existieren in der Prozessindustrie gewisse Randbedingungen, die Einfluss auf die Übertragungsphysik haben können, die es zu berücksichtigen gilt, wie zum Beispiel der Explosionsschutz. Daher muss es bei einer Implementierung von Ethernet für die Prozessindustrie einen einheitlichen und offenen Physical Layer geben.

Darüber hinaus bietet Ethernet vom Design her bereits die Möglichkeit, mehrere Kommunikationsprotokolle parallel zu nutzen. Daher bestünde theoretisch die Möglichkeit, dass jeder Hersteller sein eigenes Protokoll verwendet. Da aber in der Prozessindustrie in der Regel Feldgeräte und Leitsysteme verschiedener Hersteller zum Einsatz kommen, müssen diese Kommunikationsteilnehmer ein gemeinsames Protokoll sprechen. In der Prozessindustrie sind zwei Protokolle weltweit am weitesten verbreitet, Profinet und Ethernet IP ([10], CPF2/2 und CPF3/5). Aus diesem Grund müssen alle Kommunikationsteilnehmer beide Protokolle vollumfänglich unterstützen.

Des Weiteren sollte eine entsprechende Netzwerkstruktur so flexibel sein, dass technologische Weiterentwicklungen integriert werden können. Eine parallele Nutzung von älteren und neuen Feldgeräten muss 
möglich sein, zum Beispiel, dass eine zentrale Kommunikationskomponente mit einem Feldgerät mit einer Geschwindigkeit von 1MBit/s kommuniziert und mit einem neueren Feldgerät mit 100MBit/s.

Damit die Daten als Infrastrukturdienst zur Verfügung stehen, muss die entsprechende Übertragungstechnologie vollständig automatisiert funktionieren und zur Verfügung stehen. Das bedeutet beispielsweise, dass alle kommunikationsspezifischen Einstellungen, wie Adressen, Zeitstempelung, automatisch erfolgen müssen. Darüber hinaus ist es ebenso elementar, dass die Information zur Prozessführung, die Zusatzinformation über den Prozess sowie die Diagnosedaten aller Kommunikationsteilnehmer automatisch zur Verfügung gestellt werden und vollständig automatisiert von Hostsystemen beziehungsweise berechtigten Kommunikationsteilnehmern abgerufen werden können.

\section{ZUSAMMENFASSUNG}

Die beschriebenen Möglichkeiten und Konzepte haben das Ziel, dem Anwender eine einfache, flexible und herstellerübergreifende Nutzung aller Gerätefunktionalitäten zu ermöglichen. Dazu zählen unter anderem die Beschleunigung der Inbetriebnahme durch die definierten Namur-Standardgeräteparameter und die Vereinheitlichung der Bedienoberflächen. Die Übertragungstechnologie muss entsprechend smart werden, damit die Anforderungen der Anwender nach einfachen und skalierbaren Übertragungstechnologien erfüllt werden und darüber hinaus muss die Geräteintegration basierend auf zentral gepflegten Standards erfolgen. Nur mit solchen Systemen lassen sich die Funktionen für den Anwender so nutzbar machen, dass dieser die Systeme auch einsetzen will.

\section{REFERENZEN}

[1] IEC 61987: Industrial-process measurement and control - Data structures and elements in process equipment catalogues. IEC 2006, www.iec.ch

[2] IEC 61360: Standard data elements types with associated classification scheme for electric items. IEC 2009 , www.iec.ch

[3] NE 131: NAMUR-Standardgerät - Anforderungen an Feldgeräte für Standardanwendungen. Namur 2009

[4] NE 107: Selbstüberwachung und Diagnose von Feldgeräten. Namur 2006

[5] NE 43: Vereinheitlichung des Signalpegels für die Ausfallinformation von Digitalen Messumformern mit analogem Ausgangssignal. Namur 2003

[6] FF 908 FS 1.0: Flow Transducer Block Final Specification. Fieldbus Foundation 2012, www.fieldbus.org

[7] Profibus. Profile for Process Control Devices. 2009, www.profibus.com

[8] HCF_SPEC-160: Device Families Command Specification. Hart Foundation 2011, www.de/hartcomm.org

[9] FDI-2080: FDI Usability Style Guide. FDI Cooperation 2014, www.fdi-cooperation.com

[10] IEC61784-2: Industrial communication networks - Profiles - Part 2: Additional fieldbus profiles for real-time networks based on ISO/IEC 8802-3. IEC 2014, www.iec.ch

[11] Schlichtmann, T.: Plant Asset Management Smarte Konzepte schaffen neue Werte. Vortrag NAMUR-Hauptsitzung 2015

\section{AUTOREN}

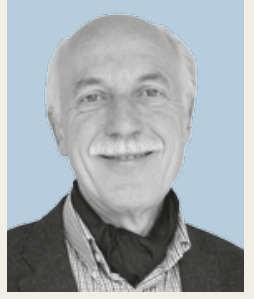

Dr. ARMIN BRUCKER (geb. 1958) ist seit seiner Promotion in experimenteller Kernphysik in der BASF SE tätig. Er war im Bereich der PLT-Planung und Betreuung von Produktionsanlagen am Standort Ludwigshafen beschäftigt, danach übernahm er lange Jahre als Senior Engineering Manager die Planung und Errichtung von Chemieanlagen. Seit 2014 leitet er im Fachzentrum PLT Feldgeräte den Bereich Durchfluss-und Temperaturmesstechnik. Er leitet den VDI/VDE GMA FA 2.40 „Durchflussmesstechnik“ und in der NAMUR den Arbeitskreis 3.2 „Durchflussmesstechnik“.

BASF SE, GTG/EI - L440,

D-67056 Ludwigshafen,

Tel. +49 (0) 62160565 64, E-mail: armin.brucker@basf.com

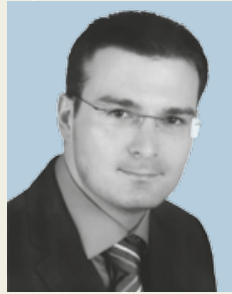

OLIVER WEIGEL (geb. 1976) ist seit seinem Studium der Automatisierungstechnik 2004 in der BASF SE tätig. Er war im Bereich der Technischen Fachzentren mit dem Schwerpunkt Evaluierung und Qualitätssicherung für Prozessleitsysteme und Bustechnik tätig. Seit 2009 arbeitet er im Bereich der globalen Planung und Errichtung von Chemieanlagen. Er ist Mitglied im NAMUR-Arbeitskreis 2.6 „Feldbus“. 